\title{
Intra-arterial transplantation of neural stem cells improve functional recovery after transient ischemic stroke in adult
}

\section{rats}

\author{
Jalali Kondori $\mathrm{B}^{1,2}$, Asadi $\mathrm{MH}^{1}$, Bahadoran $\mathrm{H}^{1}$, Yari $\mathrm{A}^{3}$, Raouf Sarshoori $\mathrm{J}^{1}$ \\ Department of Anatomy, Faculty of Medicine, Baqiyatallah University of Medical Sciences, Tehran, Iran. \\ bahmanjalali2010@gmail.com
}

\begin{abstract}
OBJECTIVES: The neural stem cell transplantation has been proposed as alternative therapy to promote functional recovery after various neurological disorders. The aim of this study was to evaluate the effect of intra-arterial transplantation of adult neural stem cells on improving local brain ischemia injuries.

MATERIALS AND METHODS: In this study, 32 male Wistar rats were used. Ischemia was induced using a middle cerebral artery obstruction with monofilament nylon suture. Neural stem cells were isolated from subventricular zone of the rat brain. 24 hours after local ischemia, the cells were labeled with Dil and transplanted intra-articularly. Evaluation of neurological movement deficits was performed using a neurological deficit score. The transplanted neural stem cells differentiation into neurons and astrocytes was assessed by immunohistochemistry.

RESULTS: The results indicated that lesion volumes in the ischemic control, PBS and the treatment groups were 31.5, 29.8 and $14.7 \%$ respectively. Our results also showed that the number of eosinophilic neurons and also neurological impairment in the treatment group was significantly reduced compared to the control and PBS groups.

CONCLUSION: The results suggested that intra-arterial transplantation of neural stem cells 24 hours after ischemia, led to a decrease in the volume of brain ischemic lesion and improved neurological outcomes (Fig. 7, Ref. 23). Text in PDF www.elis.sk. KEY WORDS: ischemic stroke, cell transplantation, subventricular zone, neural stem cells.
\end{abstract}

\section{Introduction}

Brain ischemia is one of the most common causes of mortality in human societies. It leads to neurological disorders and neuropsychological deficits such as: cognitive dysfunction, amnesia and impaired spatial learning and memory (1). Stem cells are very useful to reduce the complications of this disease. Studies showed that neural stem cells (NSCs) had a high potential for treatment of central nervous system disorders, such as cerebral ischemia (2). Methods of stem cells transplantation to the damaged tissue include intracerebral, intravenous and intra-arterial transplantation. Intravenous methods have been widely welcomed due to their ease and non-invasiveness. The use of intra-arterial transplantation methods did not have problems with the intravenous method, such as the dispersion of cells in other organs, and more of these cells reached the damaged tissue $(3,4)$. Recent studies showed that the use of intra-arterial transplantation of stem cells

${ }^{1}$ Department of Anatomy, Faculty of Medicine, Baqiyatallah University of Medical Sciences, Tehran, Iran, ${ }^{2}$ Marine Medicine Research Center, Baqiyatallah University of Medical Sciences, Tehran, Iran, and ${ }^{3}$ Department of Anatomy, Faculty of Medicine, Alborz University of Medical Sciences, Karaj, Iran Address for correspondence: B. Jalali Kondori, Department of Anatomy, Faculty of Medicine, Baqiyatallah University of Medical Sciences, Tehran, Iran. has had a significant impact on the treatment of many central nervous system diseases (5).

Adult neuronal stem cells have a high potential for treating cerebral ischemia in contrast to mesenchymal stem cells due to their inherent nervous nature. These cells produce useful neurotrophic factors, such as GDNF and NGF, which protect the glutamate-induced cellular toxicity and increase the survival of damaged motor neurons (6). In addition, it has been proven, that the secretion of valuable factors such as: VEGF from neural stem cells transplanted into the brain ischemia model has a significant effect on reducing the delayed death of nerve cells in the ischemic region $(7,8)$. In addition, the differentiation of NSCs within the neural tissue leads to the production of new neuronal and glial cells.

The purpose of this study was to evaluate the effect of intraarterial transplantation of NSCs on improving the neurological functional recovery and reduction of infarction volume caused by focal cerebral ischemia in rats. Furthermore, we determined the differentiation of transplanted NSCs in the ischemic region.

\section{Materials and Methods}

Animals

Adult male Wistar rats were purchased from Pasteur institute of Iran. During the experiment, they were kept in standard conditions 
of 12 hours of light and darkness, humidity, $25^{\circ} \mathrm{C}$ and free access to water and food. All experiments were carried out according to the ethical rules for working with animals approved by the Research Council of the Baqiyatallah university of medical sciences.

\section{Isolation of NSCs from the subventricular zone}

In order to isolate the adult NSCs, 6 to 8 weeks old rats were first anesthetized with ketamine and xylacin and after the cervical dislocation, the brain was removed, immediately transferred to the culture medium. After removing the olfactory bulbs, a coronal section was made in the optic chiasma. The subuntericular zone of the two sides was carefully separated and enzyme digestion was performed using trypsin under the laminar hood.

NSCs were cultured and expanded using neurosphere assay (9). In this method, the isolated cells were cultured in DMEM/ F12 medium containing GlutaMAX without serum. $1 \mu \mathrm{L} / \mathrm{ml}$ of $0.2 \%$ heparin, $10 \mathrm{ng} / \mathrm{ml}$ of basic Fibroblast Growth Factor F (bFG), $20 \mathrm{ng} / \mathrm{ml}$ of Epidermal Growth Factor (EGF) and $2 \%$ B27 was added to the above culture medium. Cells were cultured in incubator at $37{ }^{\circ} \mathrm{C}, \mathrm{CO}_{2} 5 \%$ and appropriate humidity. Cell viability was determined by trypan blue staining and the viability was greater than $96 \%$.

\section{Identification of neural stem cells}

An immunocytochemistry method was used to identify isolated NSCs. Accordingly, cells were cultured on poly-L-lysine-coated slides. Parafirmaddehyde $4 \%$ was used to fix the cells. The slides were incubated overnight with anti-nestin (SantaCruz, CA; $1: 100$ ) and anti-NF200 (Chemicon; 1: 200) mouse antibodies. Then they finally incubated with FITC-conjugated secondary antibody and exami ned under the fluorescent microscope.

\section{Intraluminal middle cerebral artery occlusion (MCAO)}

In this study, the intraluminal MCAO method was used to create a local ischemic model in rat brain. The 3-0 Ethicone nylon suture was used. Under general anesthesia ( $2.5 \%$ isoflurane), the skin cut was made at a distance of $2 \mathrm{~mm}$ from the midline of the neck. Common carotid, internal and external carotid arteries were observed. The Vagus nerve was carefully removed. Occipital artery and superior thyroid artery, the branches of the external carotid artery, were carefully blocked. The internal and common carotid arteries were blocked using a micro clamp. The filament was directed into the internal carotid artery via external carotid artery to deal with the resistance and block the middle cerebral artery. After MCAO for 60 minutes, the filament was withdrawn and reperfusion was performed. During the period of ischemia, the body temperature of the animal was checked and kept constant at $37^{\circ} \mathrm{C}$.

\section{Intra-arterial NSC transplantation}

Prior to transplantation of neural stem cells in the brain ischemic rats, these cells were labeled with DiI at a final concentration of $5 \mu \mathrm{g} / \mathrm{ml}$ and incubated at $37{ }^{\circ} \mathrm{C}$ for 15 minutes. The labeled cells were floated in sterilized PBS and they became ready for transplantation. 24 hours after MCAO, neuronal stem cells were transplanted. For this purpose, the rats were anesthetized using isoflurane and the CCA and ICA arteries were temporarily blocked using $6-0$ silk suture. $5^{*} 10^{5}$ floating cells in $100 \mu \mathrm{l}$ of PBS were prepared for injection with G31 syringe. The syringe containing the labeled cells entered the remaining ECA artery lumen. After fixing it with 6-0 silk suture and micro-clamps, the blocked pathway of the ICA artery was opened. The cells were injected using a micro-pump for 2 minutes. Then the distal end of the ECA was blocked with suture and blood circulation to the brain was established.

\section{Neuro-motor dysfunction test}

Animals were evaluated for ischemic motor disorders 7 days after cell transplantation. The Neurological Deficit Score (NDS) test, previously described by Plesnila et al (10), was used. The 5-point scale scoring is as follows: 1 = normal motor function, 2 $=$ Failure to extend forepaw, when suspend vertically by its tail, $3=$ Circling to the contralateral side while starting to move, $4=$ Loss of righting reflex and $5=$ No spontaneous motor activity.

\section{Histological analyses}

Measurement of the lesion volume

TTC was used to measure the ischemic lesion volume. This type of staining is used to separate metabolically active from inactive tissues. In healthy tissues, the white TTC is reduced to reddish TPF through the dehydrogenase enzyme activity. In the ischemic zone, because of the denaturation of the dehydrogenase enzymes, this reaction has not been performed and this zone is not red. In this method, the animal was first anesthetized using the ether and the brain was removed. Then, using Brain Matrix, coronal sections with a thickness of $2 \mathrm{~mm}$ were prepared. The sections were immediately placed in TTC $2 \%$ solution at $37^{\circ} \mathrm{C}$ for 20 minutes. Then, the sections were placed in buffered formaldehyde $4 \%$ for fixation of the tissue. Finally, a separate image was made from each section and the lesion volume was calculated using the NIH image analysis software.

\section{Hematoxylin and eosin staining}

7 days after cell transplantation, the animal was anesthetized using ether and intra-cardiac perfusion was performed for the initial tissue fixation. Then, the brain was removed, and a complete fixation was performed in parafarmaldehyde. The tissues were further processed using a tissue processor. In histological studies, $5 \mu \mathrm{m}$-thick sections of the tissue were cut as serial sections. H \& E staining was done with standard protocol and appropriate images were prepared.

\section{Nissl staining}

To count the number of cells and its changes, after induction of ischemia, Cresyl Violet Acetate $1.0 \%$ staining was used. The tissue sections were stained with CresylViolet and cell count was done in microscope field of view in 5 different zones of the ischemic hemisphere for all the groups. Neurons that were severely acidophilic and had a dense nucleus, were considered as eosinophilic neurons. 

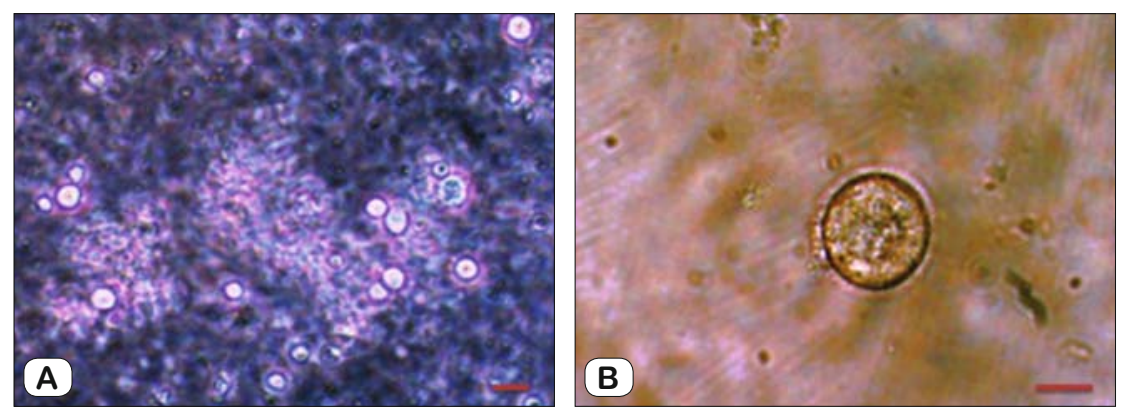

Fig. 1. Phase contrast image of the formation of the neurospheres within 5 days after the start of cultivation (A) with a magnification of 200. A mature norosphere appears seven days after the first passage with a magnification of 400 (B). Scale bar $=100 \mu \mathrm{m}$.

\section{Immunofluorescent assessment}

For immunohistochemistry, each coronal section was placed in a blocking solution (a mixture of $10 \%$ normal serum and 0.3 $\%$ Triton X-100 in PBS) and incubated for 60 minutes. Then each section was treated with specific antibodies: a neuronal nuclear antigen (NeuN for neurons; diluted in $1: 100$; Santa Cruz); an astrocytic marker (GFAP; diluted in $1: 200$; Abcam); a neural stem cell marker nestin (diluted in $1: 1000 ;$ Abcam). Finally, incubated with FITC-conjugated secondary antibody at a concentration of 1/50 for 60 minutes, and after washing with PBS, they were examined under the fluorescent microscope.

\section{Statistical analyses}

The results were presented as the mean \pm standard error mean (Mean \pm SEM). SPSS software and Student T-test were used to compare the obtained data. Non-parametric Mann-Whitney test was used for data that did not have a normal distribution. Significant difference at the level of $\mathrm{p}<0.05$ was considered for all the tests.

\section{Results}

As shown in Figure 1, the early neurospheres gradually became apparent after 5 days. By replacing part of the culture medium at this stage, the colony count increased rapidly, reached about 150 to 200 micrometers within 7 to 10 days and became ready for passage. In the second passage, the purity of the neurospheres and the number of the produced colonies increased.
Anti-nestin antibodies were used as a neural stem cell marker using immunocytochemistry. Anti-nestin antibody expression revealed that the isolated cells were neural stem cells. Anti-NF200 antibodies, which represent mature neurons, were also used as a negative control in this study (Fig. 2).

Reduction of neurological deficits after intra-arterial NSCs transplantation

24 hours after reperfusion, motor impairment was observed in the ischemic, PBS and treatment groups. The neurological score in these groups was significantly higher than in the sham group. A week after intra-arterial transplantation of NSCs, motor disorders were significantly reduced in the treatment group and the mean scores of NDS in the treatment group were significantly lower than that of the ischemic and PBS groups (Fig. 3).

\section{Transplantation of NSCs reduces the infarct volumes}

The results of TTC method showed that in the treatment group, the transplantation of NSCs reduced the ischemic lesion in the rat's brain. Quantitative analysis of the mean volume of ischemic lesion indicates a significant reduction in ischemic lesion volume in the treatment group compared with the ischemia control and PBS groups (Fig. 4).

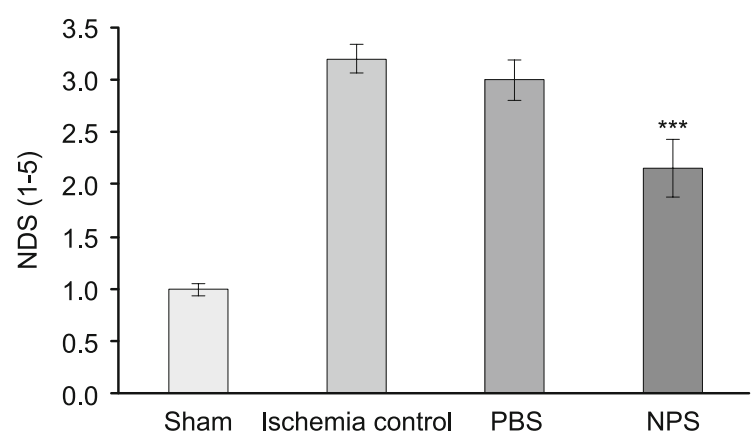

Fig. 3. Neurological dysfunctions scoring in the sham, ischemia control, PBS and treatment groups one week after the cell transplantation. The data are displayed as the mean \pm SEM. $* * *$ indicates a significant difference between the treatment group with ischemia control and PBS groups at $\mathbf{p} \leq \mathbf{0 . 0 0 1}$.
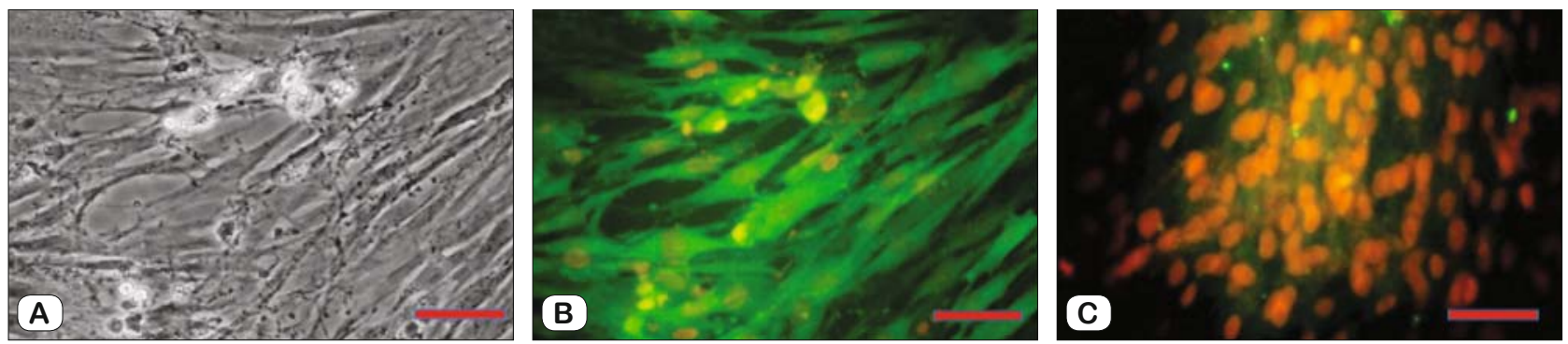

Fig. 2. Phase contrast image with a 200x magnification of NSCs (A), expression of nestin in NSCs (B), and non-expression of the NF200 marker as a negative control in these cells; Scale bar $=100 \mu \mathrm{m}$. 


$$
\text { A }
$$

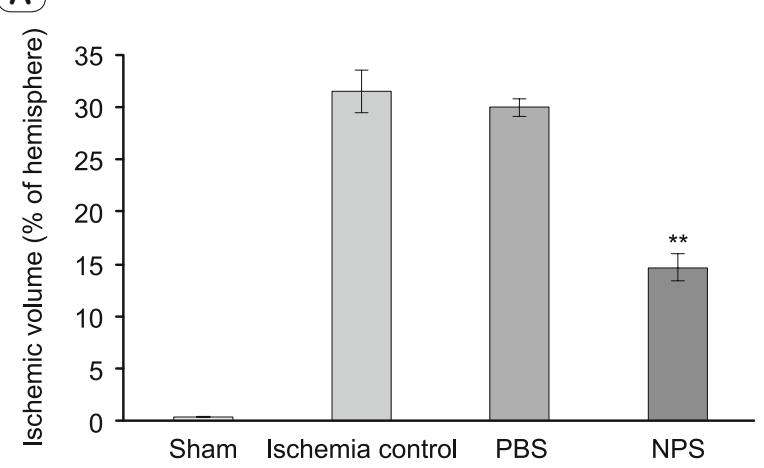

B

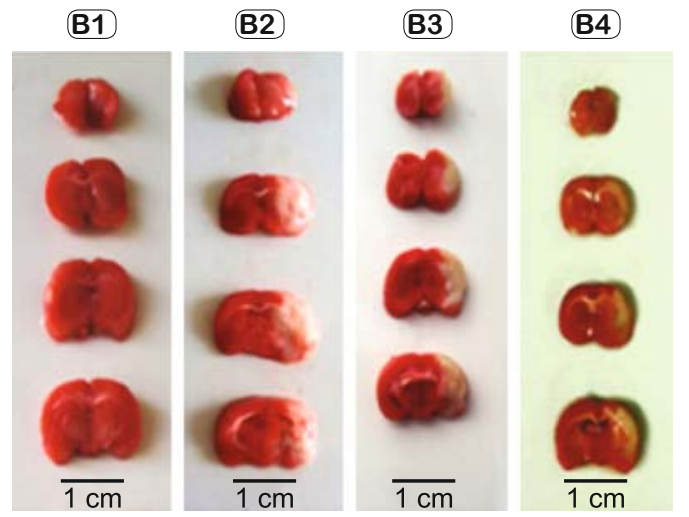

Fig. 4. (A) The comparison of the volume of ischemic lesion between different groups is shown. **: Significant difference was observed in the treatment group with ischemia control and PBS groups at $p<0.01$. (B) TTC staining to show the onset of ischemia in the rat's brain. (B1) shamgroup, (B2) control of ischemia, (B3) PBS group and (B4) treatment. White areas indicate an ischemic lesion.
Transplantation of NSCs reduces cellular damage in the ischemic zone

The results of histological studies indicated that the death of neural cells in the treatment group was lower than that of the ischemia control and PBS groups. As shown in Figure 5, in hematoxylin and eosin staining, morphology of the neurons and their nuclei were the criteria for diagnosis of cell damage. Accordingly, healthy neurons had a clear cytoplasm and nucleus, but the degenerated neurons had a dark cytoplasm and dense nucleus.

Cresyl violet staining results showed that the number of cells in the ischemic zone was significantly lower than that of the ischemia control and PBS groups. Moreover, damaged neurons with shrink, dense and unclear nucleus are observed in the ischemic zone. In the treatment group, the number of damaged cells decreased compared to the other groups. In the sham group, no pathological changes were observed (Fig. 6).

Transplanted NSCs differentiate into neurons and astrocytes in vivo

The results of the immunohistochemistry method showed that the intra-arterial transplanted cells are homed in the ischemic zone. As shown in Figure 7, the merged images indicated that DiI labeled NSCs differentiated into both neuron and astrocyte cells.

\section{Discussion}

The results of the present study showed that the neuro-motor disorders caused by ischemia decreased significantly in the group that received NSCs transplantation 24 hours after reperfusion. This may be due to the homing of this cells in the ischemic zone and cellular communication with damaged cells that have not yet been eradi-
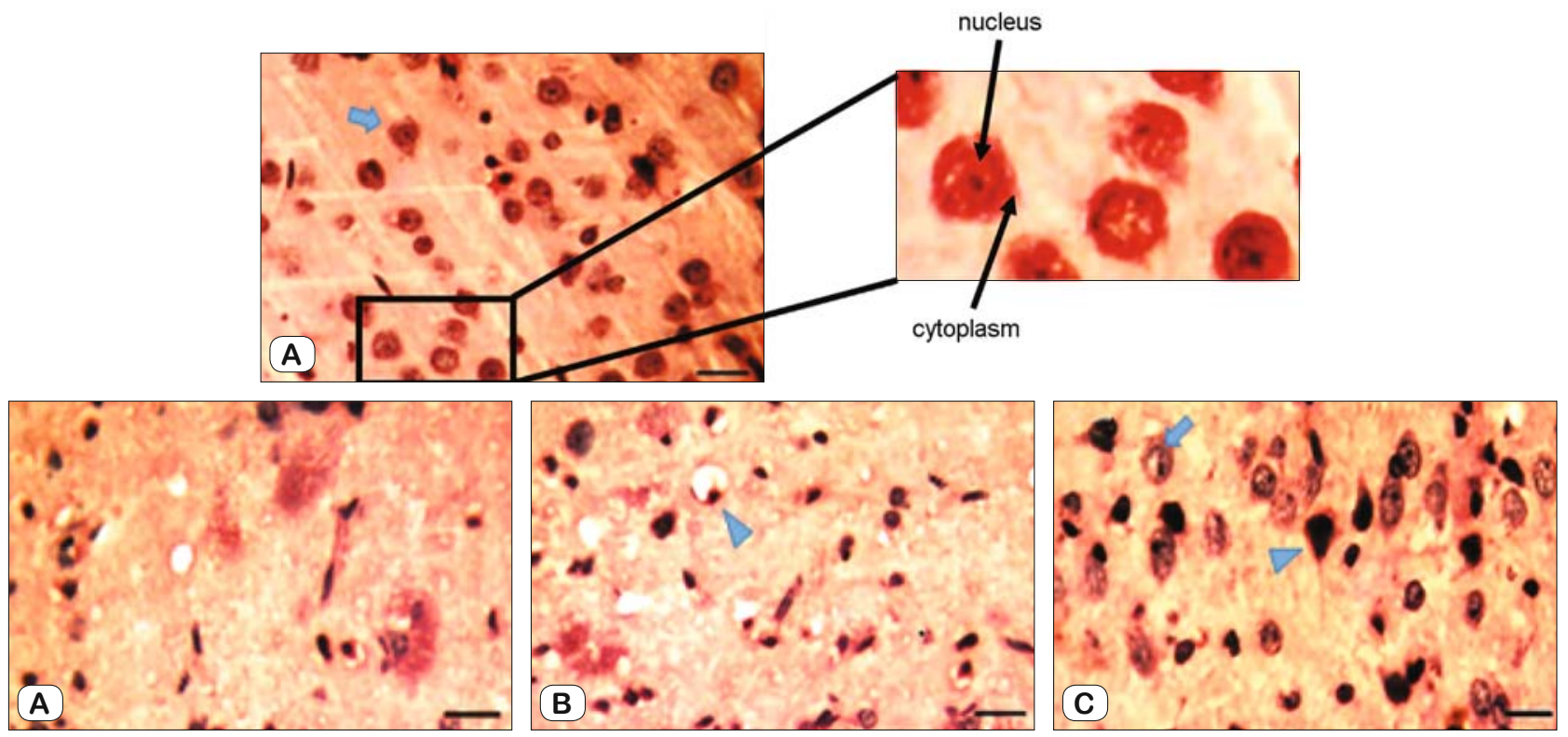

Fig. 5. Hematoxylin-eosin stained sections in sham group (A), ischemic control group (B), PBS group (C) and treatment group (D) with 400x magnification. In the treatment group, the number of cells with dark cytoplasm and dense nucleus (tip of the arrow) was significantly reduced compared to the ischemic control and PBS groups. Normal neurons with clear cytoplasm and nucleus are shown with arrows. Scale bar $=\mathbf{5 0} \mu \mathrm{m}$. 

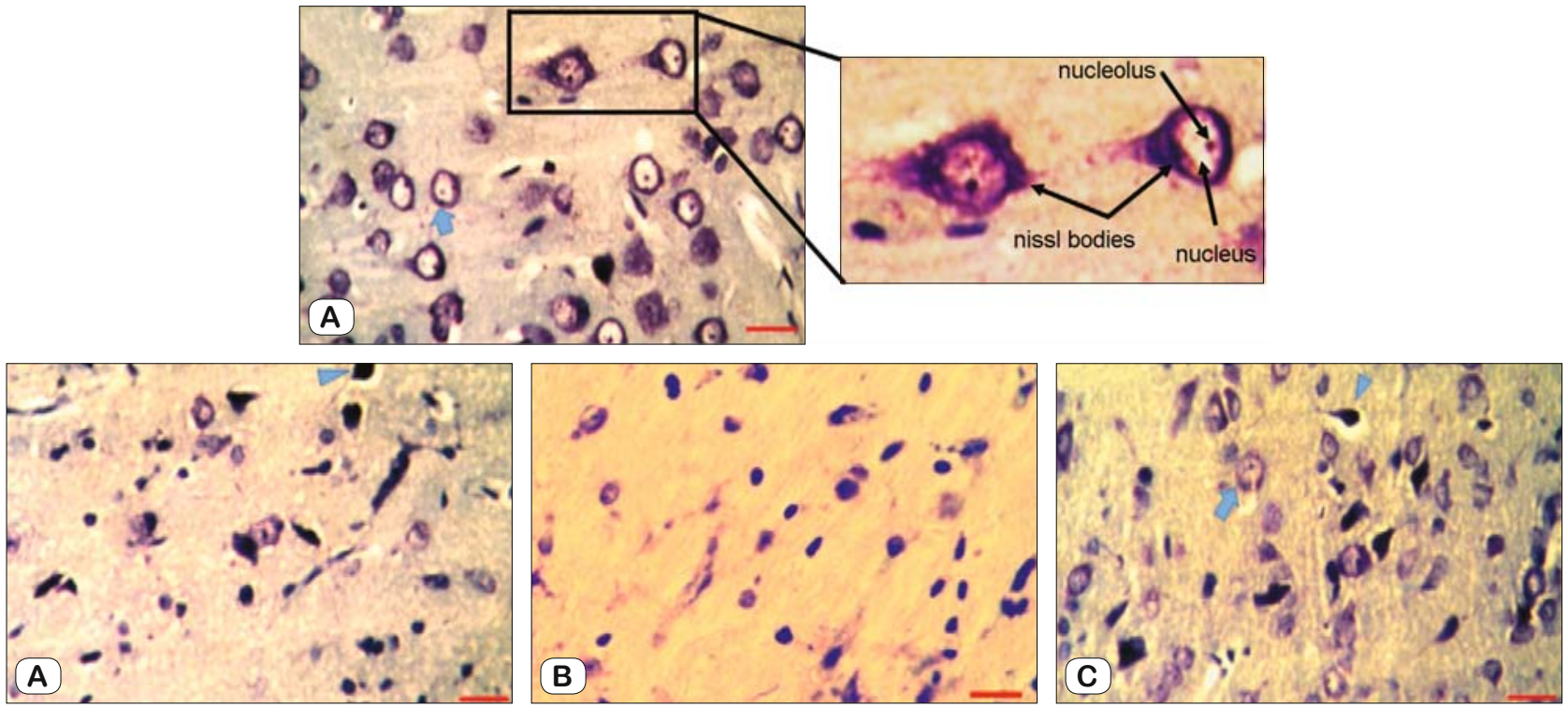

Fig. 6. Cresyl violet stained sections in control group (A), ischemia control group (B), PBS group (C) and treatment group (D) with 400x magnification. In the treatment group, the number of cells with dark cytoplasm and dense nucleus (tip of the arrow) was significantly reduced compared to the ischemic control and PBS groups. Normal neurons with a clear nucleus are shown with arrows. Scale bar $=\mathbf{5 0} \mu \mathrm{m}$.
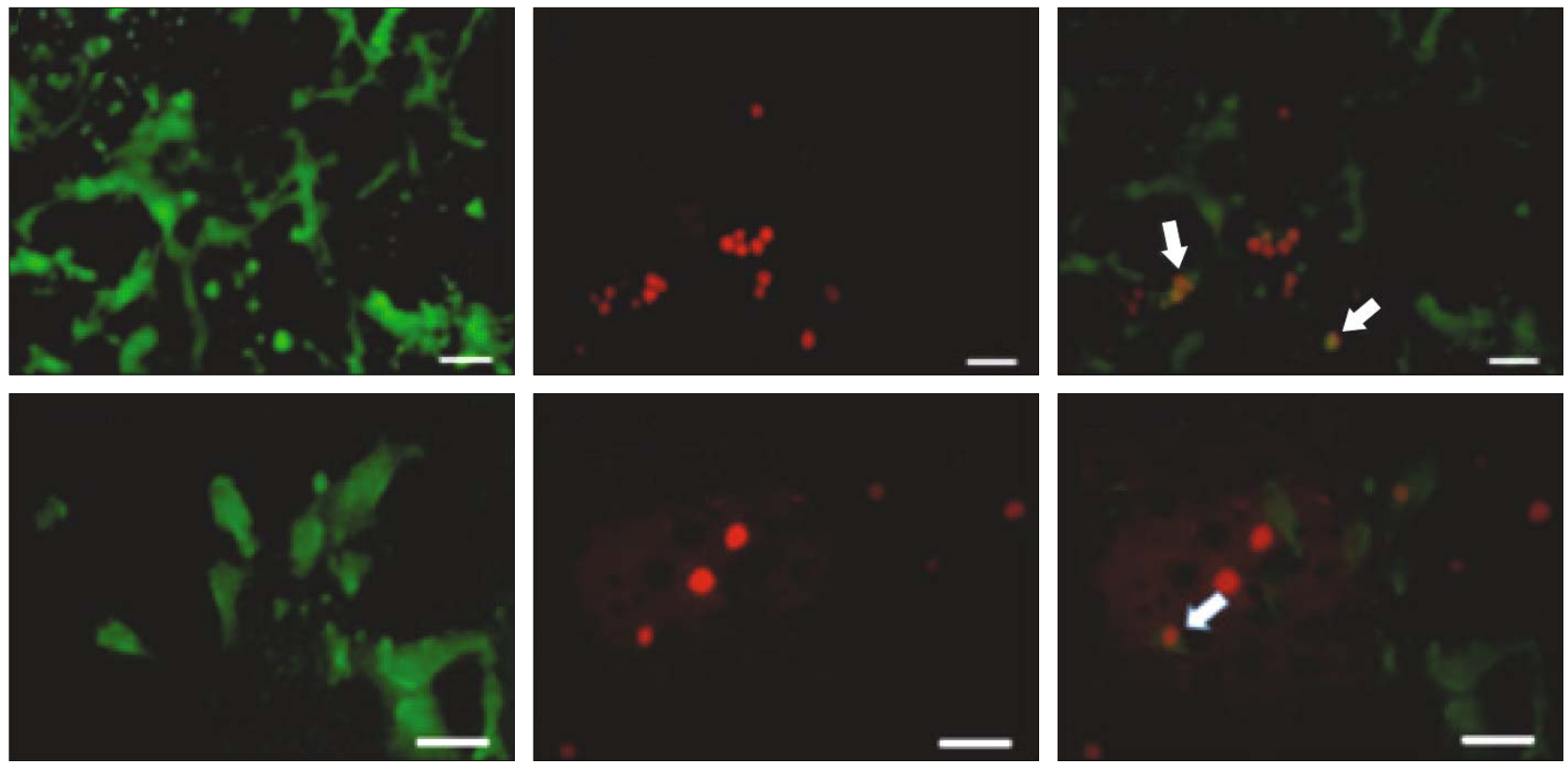

Fig. 7. Immunohistochemistry results indicated that DiI labeled NSCs differentiated into both neuron and astrocyte cells. The GFAP and Neu $\mathrm{N}$ magnifications are 100 and 200x respectively.

cated. Our results showed, that these cells differentiated into astrocytes and neurons in the ischemic zone. The differentiation of these cells into astrocytes may support and improve the damaged cells.

Our results also indicated that the volume of ischemic region in the treatment group was smaller than the ischemic control and PBS groups. These results indicated that the number of damaged nerve cells in the group receiving the NSCs was lower than the ischemia control and PBS groups. In the similar study done by Cheng et al (2015), NSCs were administered by intravenous injec- tion in ischemic model mice (11). This study also confirmed similar results. According to the findings of this study, transplantation of neural stem cells improved symptoms of neuro-motor disorders. Accordingly, the transplanted NSCs, in addition to maintaining reproductive power in the in vitro environment, induced proliferation in endogenous cells.

Several studies were conducted on the isolation of NSCs from the brain of the mouse. In these studies, the brains of several mice have been used simultaneously to isolate and differentiate NSCs 
$(12,13)$. In the present study, these cells were isolated from subventricular zone of a single adult rat. Although the use of a single rat brain to isolate these cells is much more difficult and requires the microdissection and accurate enzymatic digestion, implementation of this protocol on a brain allows the application of this protocol in limited cases such as: transgenic mice or genetic studies (14).

The analysis of studies that used the intraluminal MCAO method for local ischemia in an animal model shows different results. This is due to the existence of different variables and variations in the details of the implementation of the method. The variables of previous studies included the ischemia time, weight of the studied mice, anesthesia methods, and so on. In this study, it was attempted to provide a standard and repeatable method for inducing local temporary ischemia by studying different variables. Various researchers in the intraluminal MCAO method have used 90, 60, and 120-minute intervals $(15,16)$. The results of Garcia and Li's studies showed that MCAO longer than 60 minutes caused more extensive ischemia, especially in the hippocampus and hypothalamus. Damage to hypothalamic cells caused hyperthermia in mice 24 hours after ischemia $(17,18)$. Hyperthermia caused increased cell death and animal mortality (19). In addition, studies showed that damage to the hypothalamus in human ischemia has been rarely observed (20). The animal model should be designed in such a way that damage to the hypothalamic zone is not extensive.

It is also important to note that damage to the striatum zone begins shortly after ischemia and generally causes necrosis in the cells. However, damage to the cortex begins with a delay and gradually causes apoptosis in the cell (21). Therefore, re-perfusion reduces cell damage in the cortex. In addition, hypoxia-induced genes that play a neuroprotective role, such as heat shock protein $70, \mathrm{Bcl}-2, \mathrm{Bcl}-\mathrm{XL}$, and Bax, are expressed exclusively in the cortex, and transplantation of NSCs within 24 hours after the onset of ischemia can reduce cortex cell death $(22,23)$. Due to these reasons, 60-minute $\mathrm{MCAO}$ seems to be more suitable for creating a local ischemia stroke model.

\section{Conclusion}

The results of this study showed that the transplantation of neural stem cells within 24 hours after ischemia led to a reduction in the neural cells death in the ischemic zone, and the brain damage decreased significantly and improved neuro-motor disorders.

\section{References}

1. Donnan GA, Fisher M, Macleod M, Davis SM. Stroke. Lancet 2008; 371 (9624): 1612-1623.

2. Ottoboni L, Merlini A, Martino G. Neural Stem Cell Plasticity: Advantages in Therapy for the Injured Central Nervous System. Front Cell Dev Biol 2017; 5: 52.

3. Willing AE, Lixian J, Milliken M, Poulos S, Zigova T, Song S et al. Intravenous versus intrastriatal cord blood administration in a rodent model of stroke. J Neurosci Res 2003; 73: 296-307.

4. Rodriguez JP, Coulter M, Miotke J, Meyer RL, Takemaru K, Levine JM. Abrogation of beta-catenin signaling in oligodendrocyte precursor cells reduces glial scarring and promotes axon regeneration after CNS injury. J Neurosci 2014; 34: 10285-10297.

5. Vasconcelos-dos-Santos A et al. Intravenous and intra-arterial administration of bone marrow mononuclear cells after focal cerebral ischemia: Is there a difference in biodistribution and efficacy? Stem Cell Research 2012; 9: 1-8.

6. Llado, J, Haenggeli, C, Maragakis, $\mathbf{N J}$ et al. Neural stem cells protect against glutamate-induced excitotoxicity and promote survival of injured motor neurons through the secretion of neurotrophic factors. Mol Cell Neurosci 2004; 27: 322-331.

7. Chen J, Zhang ZG, Li Y, Wang L, Xu YX, Gautam SC et al. Intravenous administration of human bone marrow stromal cells induces angiogenesis in the ischemic boundary zone after stroke in rats. Circ Res 2003; 92: 692-699.

8. Senior K. Angiogenesis and functional recovery demonstrated after minor stroke. Lancet 2001; 358-817.

9. Deleyrolle LP, Rietze RL, Reynolds BA. The neurosphere assay, a method under scrutiny. Acta Neuropsychiatr 2008; 20: 2-8.

10. Plesnila N, Zinkel S, Le DA, Amin-Hanjani S, Wu Y, Qiu J et al. BID mediates neuronal cell death after oxygen/glucose deprivation and focal cerebral ischemia. Proc Natl Acad Sci USA 2001; 98: 15318- 15323.

11. Yi Cheng, Jinjing Zhang, Liancheng Deng et al. Intravenously delivered neural stem cells migrate into ischemic brain, differentiate and improve functional recovery after transient ischemic stroke in adult rats. Int J Clin Exp Pathol 2015; 8 (3): 2928-2936.

12. Wang TT, Jing AH, Luo XY, Li M, Kang Y, Zou XL, Chen H, Dong J, Liu S. Neural stem cells: isolation and differentiation into cholinergic neurons. Neuroreport 2006; 17 (13): 1433-1436.

13. Kim JY, Lee J-H, Sun W. Isolation and culture of adult neural stem cells from the mouse subcallosal zone. J Vis Exp 2006; 118: 1-8.

14. Guo W, Patzlaff NE, Jobe EM, Zhao X. Isolation of multipotent neural stem or progenitor cells from both the dentate gyrus and sub-ventricular zone of a single adult mouse. Nature Protocols 2012; 7 (11): 2005-2012.

15. Steele EC Jr, Guo Q, Namura S. Filamentous middle cerebral artery occlusion causes ischemic damage to the retina in mice. Stroke 2008; 39 : 2099-2104.

16. Thomas CS. Rodent models of focal stroke: Size, mechanism and purpose. NeuroRx 2005; 2: 396-409.

17. Garcia JH, Liu KF, Ho KL. Neuronal necrosis after middle cerebralartery occlusion in Wistarrats progresses at different timeintervals in the caudoputamen and the cortex. Stroke 1995; 26: 636-642.

18. Fluri F, Schuhmann MK, Kleinschnitz C. Animal models of ischemic stroke and their application in clinical research. Drug Des Devel Ther 2015; 9: 3445 .

19. Reglodi D, Somogyvari-Vigh A, Maderdrut JL, Vigh S, Arimura A. Postischemic spontaneous hyperthermia and its effects in middlecerebral artery occlusion in the rat. ExpNeuro 2000; 163: 399-407.

20. Carmichael ST. Rodent models of focal stroke: size, mechanism, and purpose. NeuroRx 2005; 2 (3): 396-409.

21. Radak D, Katsiki N, Resanovic I, Jovanovic A, Sudar-Milovanovic E, Zafirovic $\mathbf{S}$ et al. Apoptosis and acute brain ischemia in ischemic stroke. Curr Vasc Pharmacol 2017; 15 (2): 115-122.

22. Yokota C, Kaji T, Kuge Y, Inoue H, Tamaki N, Minematsu K. Temporal and topographic profiles of cyclooxygenase-2 expressionduring $24 \mathrm{~h}$ of focal brain ishemia in rats. Neurosci Lett 2004; 357: $219-222$.

23. Kim JY, Han Y, Lee JE, Yenari MA. The 70-kDa heat shock protein (Hsp70) as a therapeutic target for stroke. Expert Opin Ther Targets 2018; 22 (3): 191-199.

Received July 17, 2019. Accepted October 3, 2019. 